EPJ Web of Conferences 37, 06012 (2012)

DOI: $10.1051 /$ epjconf/20123706012

(C) Owned by the authors, published by EDP Sciences, 2012

\title{
Double Pion Photoproduction off nuclei - search for the in-medium modification of the $\sigma$-meson
}

\author{
Yasser Maghrbi ${ }^{a}$ for the A2 Collaboration \\ Department of Physics, University of Basel, CH-4056 Basel, Switzerland
}

\begin{abstract}
The photoproduction of $\pi^{0} \pi^{0}$ and $\pi^{0} \pi^{+/-}$pairs has been investigated in view of the of in-medium modifications the $\sigma$-meson. The measurements have been done at the Mainz MAMI accelerator, using the Glasgow photon tagging spectrometer and a $4 \pi$ electromagnetic calorimeter, consisting of the Crystal Ball and TAPS detectors . The measurements covered different nuclear targets $\left({ }^{2} \mathrm{H},{ }^{7} \mathrm{Li},{ }^{12} \mathrm{C},{ }^{40} \mathrm{Ca}\right.$, and $\left.{ }^{n a t} \mathrm{~Pb}\right)$ and the statistical quality of the data was improved compared to the previous experiments. The energy and mass dependence of the invariant mass distributions suggests that Final State Interactions play a very important role, but some of the observed effects can also be related to a possible in-medium modification of the $\sigma$-meson.
\end{abstract}

\section{Introduction}

The properties of hadrons in nuclear matter is a much discussed topic in nuclear physics as it is related to a regime where the Quantum-Chromo Dynamics (QCD) is not accessible to perturbative methods. At low energies the spontaneous breaking of the chiral-symmetry occurs and without it, hadrons would appear as mass degenerate parity doublets. This symmetry breaking can be seen in the hadron spectrum and in particular for the $\sigma$-meson. The mass split between the $\sigma$ and the pion, its chiral partner is of about $600 \mathrm{MeV}$.

A partial symmetry restoration at high density or temperature can be however possible according to many models. The restoration of the symmetry is related to the temperature and density dependence of the quark condensate which is indirectly connected to the in-medium properties of hadrons via QCD sum rules. One of the most dicussed signatures of such an effect is the degeneresene of the $\sigma$-meson and the pion at a certain limit[1]. An effect on the invariant mass distribution of pion pairs is therefore expected to be seen since the $\sigma$-meson couples strongly to such iscoscalar pairs.

First effects have been observed by the CHAOS collaboration in pion-induced reactions [2] and later by the CB@BNL collaboration [3]. Both experiments observed an in-medium modification of the $\pi \pi$ interaction in the $I=J=0$ channel. A shift towards small invariant masses with increasing nuclear density was observed in the $\pi^{+} \pi^{-}$channel (resp. $\pi^{0} \pi^{0}$ for CB@BNL) but not in the $\pi^{+} \pi^{+}$(resp. $\pi^{0} \pi^{+/-}$for CB@BNL). More recently, a shift of the neutral channel spectra increasing with atomic mass number have been observed at TAPS experiment at MAMI (comparison of carbon and lead data) [4] with no effects on the mixed charge channel. However a detailed study of the invariant mass distributions for calcium nuclei revealed that a significant part of the effect is related to the pion Final State Interactions (FSI)[5].

\section{Experimental setup and data analysis}

The main difference of this setup compared to the previous measurements with TAPS at MAMI [4,5] is the almost $4 \pi$ coverage of the solid angle and the use of the PID for the identification of charged

\footnotetext{
a e-mail: yasser.maghrbi@unibas.ch
} 

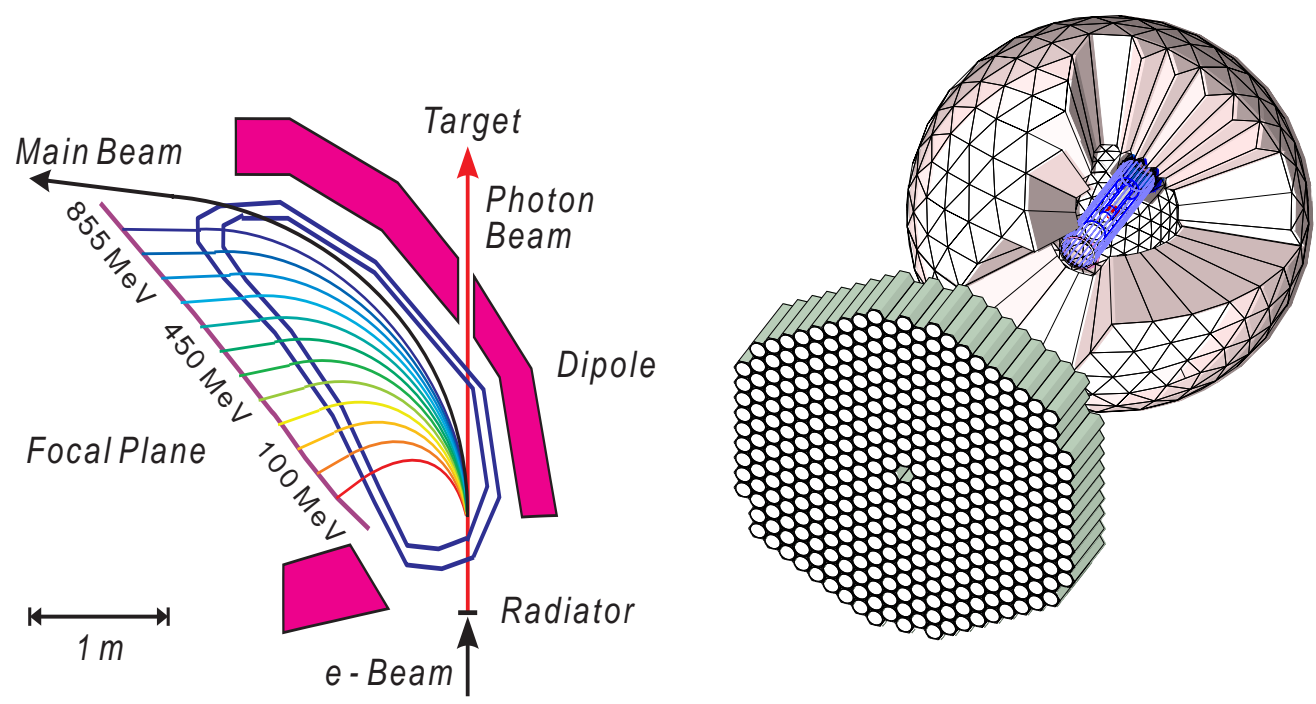

Fig. 1. General views of the detector setup. Left: Tagger, Right: the CB-TAPS detector system.

pions. The primary electron beam of $883 \mathrm{MeV}$ produced bremsstrahlung in a copper radiator of $10 \mu \mathrm{m}$ thickness. The photons were tagged with the Glasgow magnetic spectrometer with a typical resolution of $2 \mathrm{MeV}$. Irradiated were four solid-state targets $\left({ }^{7} \mathrm{Li},{ }^{12} \mathrm{C},{ }^{40} \mathrm{Ca},{ }^{n a t} \mathrm{P}\right)$ and a liquid deuterium target of similar radiation lengths.

The detector setup (Fig. 1) was an electromagnetic calorimeter combining the CrystalBall made of $672 \mathrm{NaI}$ elements with TAPS made of $510 \mathrm{BaF}_{2}$ detectors configured as a forward wall. The Ball was equipped with two additional inner detectors for the identification of the charged particles, the PID (Particle Identification Detector) and the MWPCs (Multi-Wire Proportional Chamber).

The neutral pions were identified via their decay photon pairs. The charged pions were identified via an $\Delta E-E$ analysis using CB and PID. Missing mass techniques were used to remove background from competing channels. The detection efficiency of the setup was simulated with the GEANT package.

The experimantal setup and the data analysis are described in details in $[6,7]$.

\section{Results and discussion}

Invariant mass distributions of the $\pi^{0} \pi^{0}$ and the $\pi^{0} \pi^{ \pm}$for the different targets have been extracted for the energy range from 400 to $460 \mathrm{MeV}$ and show an accumulation of strength at small invariant masses with increasing nuclear mass in both channels (Fig. 2). The effect can be better seen in Fig. 2 (right-hand side) where the composite ratios defined by

$$
C_{\pi \pi}\left(A_{1}, A_{2}\right)=\frac{\left(d \sigma\left(A_{1}\right) / d M\right) / \sigma\left(A_{1}\right)}{\left(d \sigma\left(A_{2}\right) / d M\right) / \sigma\left(A_{2}\right)}
$$

is plotted for incident photon energies from $400 \mathrm{MeV}$ to $460 \mathrm{MeV}$ with $A_{1}, A_{2}$ are nuclear mass numbers.

At this energy range, the effect is therefore certainly not related to properties of the $\sigma$-meson, which does not couple to the mixed-charge channel but is most likely due to the final state interactions.

The importance of FSI can be estimated by studying the ehavior of the total cross sections scaled by the mass number $A$ as function of incident photon energy, which can be parameterized with the scaling coefficient $\alpha$ from: 

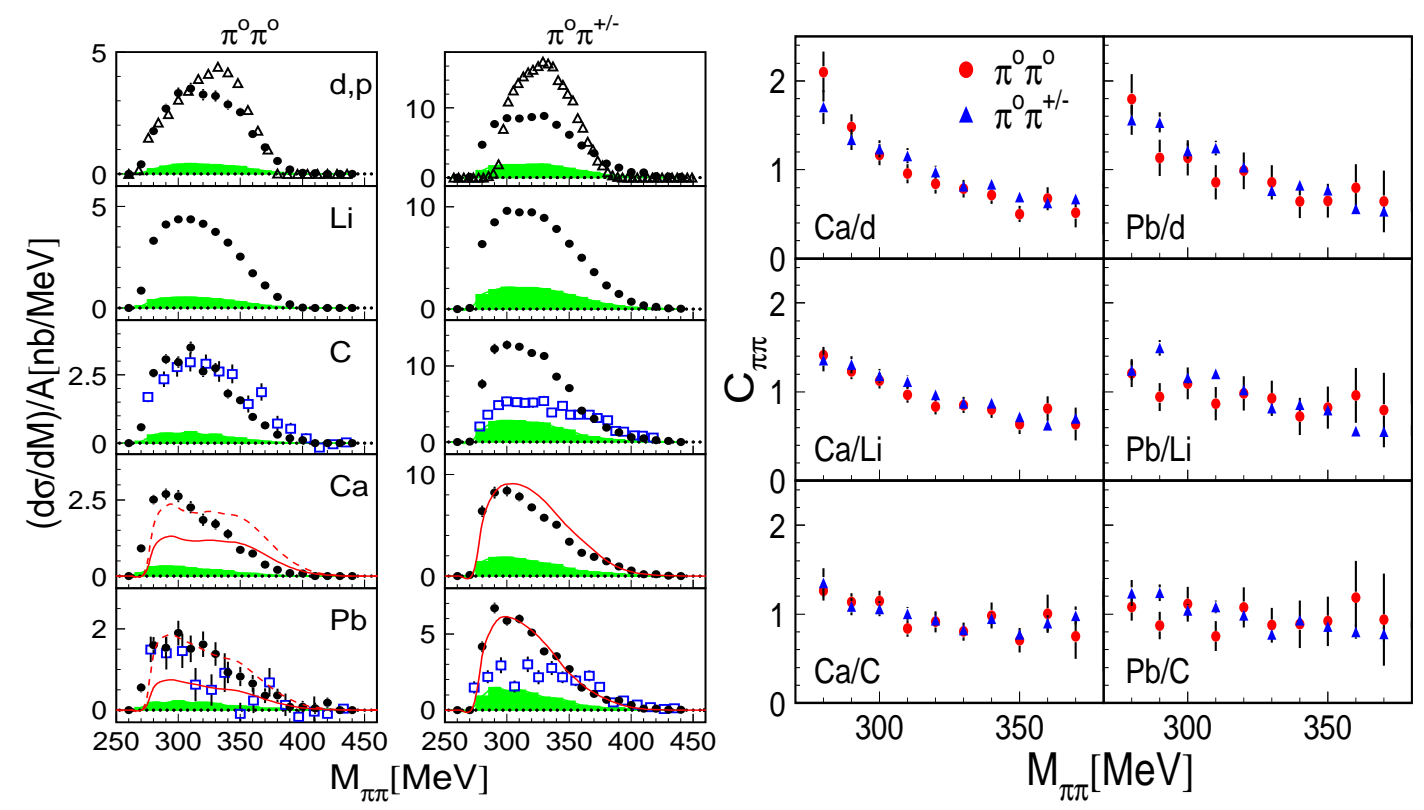

Fig. 2. Left: Invariant mass distributions of pion pairs normalized to the nuclear mass numbers for $\mathrm{E}_{\gamma}=[400-460]$ $\mathrm{MeV}$. From top to bottom for deuterium (and proton, open triangles), lithium, carbon, calcium, and lead targets. Open (blue) squares: previous data from [4]. Shaded (green) bands: systematic uncertainties. (Red) solid curves: GiBUU results [8], dashed: arbitrary rescaled for easier comparison of the shapes. Right: Composite ratios for $\pi^{0} \pi^{0}$ (red dots) and $\pi^{0} \pi^{ \pm}$(blue triangles) $\mathrm{E}_{\gamma}=$ [400-460] MeV. Left-hand side: calcium, right-hand side: lead, compared to d, $\mathrm{Li}, \mathrm{C}$.

$$
\sigma\left(A, E_{\gamma}\right) \propto A^{\alpha\left(E_{\gamma}\right)} .
$$

At incident photon energies close to the production threshold $\alpha$ is close to unity, indicating negligible losses due to pion absorption. The coefficient then approaches $2 / 3$ for beam energies between $500 \mathrm{MeV}$ and $600 \mathrm{MeV}$, indicating a strong absorption. The scaling behavior is similar for both isospin channels, so that comparable FSI effects are to be expected.

The shapes of pion-pion invariant-mass distributions for different ranges of incident photon energies are compared in Fig. 3. For both isospin channels, at the lowest incident photon energies, the distributions are similar for all nuclei. They agree with a Fermi-smeared version of the elementary cross section average of proton and neutron cross sections, represented by the deuteron data. At higher incident photon energies they start to differ, and for fixed photon energy, the heaviest nuclei have the softest distributions. This general trend can be related to the increase of FSI with rising beam energy and rising mass. It can be easily understood that FSI tends to soften the distributions. Two effects are important. Inelastic scattering tends to decrease the kinetic energy of the pions and their mean-free path increases with decreasing energy.

The residual effect observed in the neutral channel is larger than the mixed charge one, indicating a possible in-medium modification of the $\sigma$-meson. However, such modification can only be studied on top of the FSI effects, which requires detailed model descriptions of FSI.

\section{Conclusions}

Precise results have been obtained for the invariant mass distributions of $\pi^{0} \pi^{0}$ and $\pi^{0} \pi^{ \pm}$pairs produced from deuterium, $\mathrm{Li}, \mathrm{C}, \mathrm{Ca}$, and $\mathrm{Pb}$ targets from production threshold up to incident photon energies of $600 \mathrm{MeV}$. A pronounced shift of strength towards small invariant masses as function of the nuclear mass number is observed for both final states. This effect increases with increasing beam energy 

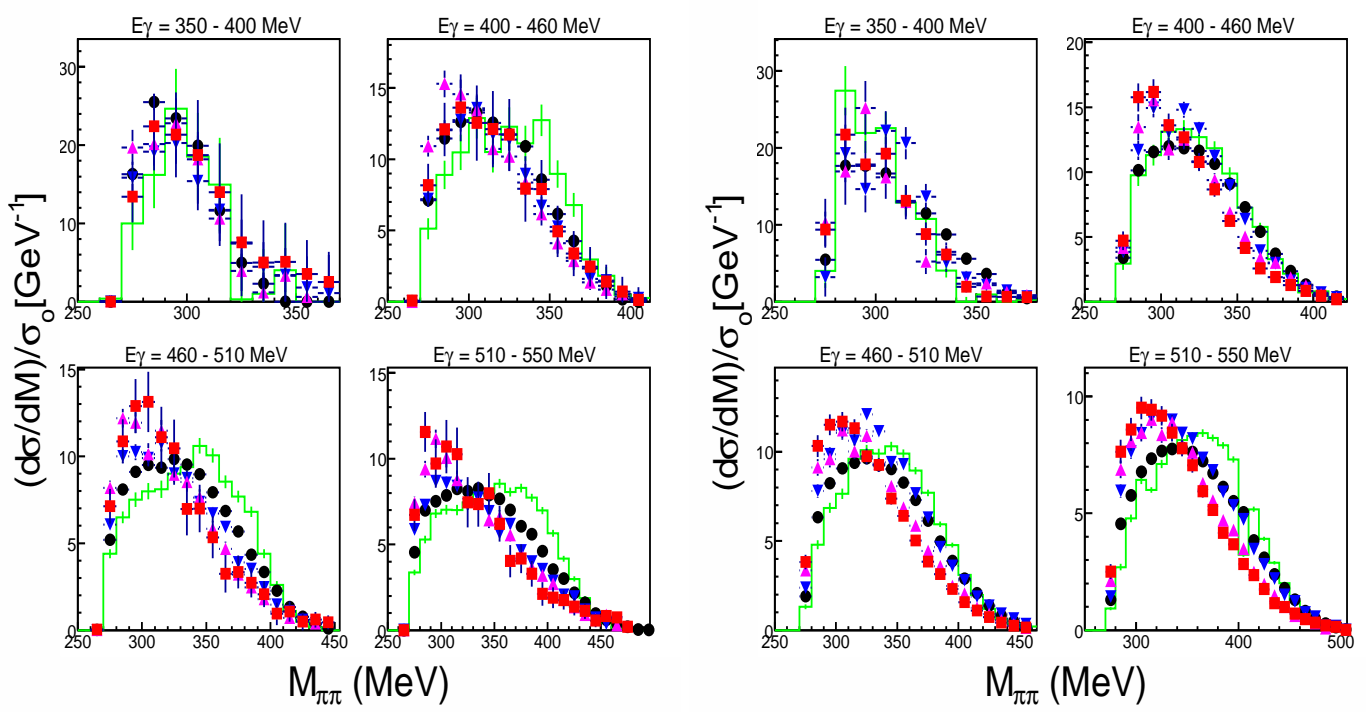

Fig. 3. Pion-Pion invariant mass distributions for different ranges of $E_{\gamma}$ normalized to the total cross sections. Left-hand part: $\pi^{0} \pi^{0}$, right-hand part: $\pi^{0} \pi^{ \pm}$. Deuteron: (green) histogram, Li (black) dots, C (blue) down triangles, $\mathrm{Ca}$ (magenta) up triangles, $\mathrm{Pb}$ (red) squares.

and can be related to FSI of the pions. However, the obseved effect is larger for the neutral channel indicating a possible modification of the $\sigma$-meson, which needs a detailed model calculation of the FSI.

\section{Acknowledgments}

This work was supported by Schweizerischer Nationalfonds and Deutsche Forschungsgemeinschaft (SFB 443).

\section{References}

1. V. Bernard et al., Phys. Rev. Lett. 59, (1987) 966

2. F. Bonutti et al., Phys. Rev. C 60, (1999) 018201

3. A. Starostin et al., Phys. Rev. Lett. 85, (2000) 5539

4. J. G. Messchendorp et al., Phys. Rev. Lett. 89, (2002) 222302

5. F. Bloch et al., Eur. Phys. J. A 32, (2007) 219

6. S. Schumann et al., Eur. Phys. J. 43, (2010) 269

7. F. Zehr et al. Eur. Phys. J. A (2012) in press

8. O. Buss et al., Phys. Rept. 512, (2012) 1 